\title{
Introduction to the Self-Quantification With Activity Tracking Technologies: Opportunities and Threats Minitrack
}

\author{
Aylin Ilhna \\ Heinrich Heine University Düsseldorf \\ aylin.ilhan@hhu.de
}

\section{Introduction}

The increasingly ubiquitous activity tracking technology, including activity trackers and mobile health applications, enables users to quantify and monitor their daily behavior. Here meant are especially the physical activities, but also general behavioral patterns like sleep duration and quality, or food intake. Researchers of different fields (computer science, information science, system sciences, engineering, medicine, and social sciences) have investigated different aspects of activity tracking technology, such as the acceptance or discontinuation of using activity trackers, the measurement accuracy of different activity trackers or the impact of the technology on the behavior of its users (substantiated with various theories, e.g., from behavioral sciences on motivation).

Activity tracking technologies (e.g., fitness tracker, mobile applications, smart watches) collect different data pieces that might be perceived as sensitive by its users [1, 2]. According to Fietkiewicz and Henkel [3], more user-centered research considering privacy-related investigations on activity tracking technologies is needed. Further, Silva and colleagues [4] point out as well that "[m]ost research focuses on methods and techniques to prevent unauthorized access of personal information in the context of mHealth."

In the past, several data breaches such as in the MyFitnessPal case [5] or the Strava case [6] showed the importance of data privacy and data security within the domain of activity and fitness tracking. This minitrack will offer insights into a study that thematized to what extent privacy assurance statements can influence satisfaction of the users and, further, how it changes after a data breach [7].

The authors of the paper entitled "Fitness First or Safety First? Examining Adverse Consequences of Privacy Seals in the Event of a Data Breach"[7] investigated the so-called privacy seals used by fitness tracking companies and their impact on continuance expectation after a data breach. The study was based

\author{
Kaja J. Fietkiewicz \\ Heinrich Heine University Düsseldorf \\ kaja.fietkiewicz@hhu.de
}

on the Expectation-Confirmation Theory (ECT) and used exemplary privacy assurance statements in order to examine the potential of mitigating any consequences of data breaches in advance, so that the continuance intention is maintained. The results indicate that the use of the privacy assurance statements might lead to high security expectations. Consequently, the failure to meed these expectations has a negative impact on satisfaction and, as a result of this, the continuance intention.

Apart from privacy-related aspects, this minitrack will also shed light on the challenges and opportunities present when integrating activity tracking technologies in specific health and therapy-related situations. In last year's minitrack on Health Information Behavior and Activity Tracking Technology Emerson and colleagues [8] already discussed workplace health promotion through integration of mobile health applications.

The second paper in this minitrack entitled "Supervised physical exercise therapy of peripheral artery disease patients: M-health challenges and opportunities" addressed a topic from the mHealth domain, in particular the development and testing of an application for supervised physical exercise therapy of peripheral artery decease patients [9]. The authors discuss the opportunities and challenges of such tools detected during the co-creation process of the mobile application. During the test phase of the application, several limitations as well as potential sources of noise in the supervision data could be detected based on the users' interaction with the tool. Paredes and colleagues [9] underline that "user interaction with the application are minimal, in some cases, just to start the recording of the exercise." This in turn impacts the quality of collected data. The study points out important challenges that arise when developing this kind of applications.

\section{References}

[1] K. J. Fietkiewicz and A. Ilhan, "Fitness tracking technologies: Data privacy doesn't matter? The (un)concerns of users, former users, and non-users," in 
Proceedings of the 53rd Hawaii International Conference on System Sciences, pp. 3439-3448, ScholarSpace, 2020.

[2] C. Lidynia, P. Brauner, and M. Ziefle, "A step in the right direction - understanding privacy concerns and perceived sensitivity of fitness trackers," in Advances in Human Factors in Wearable Technologies and Game Design. AHFE 2017. Advances in Intelligent Systems and Computing (T. Ahram and C. Falcão, eds.), vol. 608, pp. 42-53, Springer, 2018.

[3] K. J. Fietkiewicz and M. Henkel, "Privacy protecting fitness trackers: An oxymoron or soon to be reality?," in Social Computing and Social Media. User Experience and Behavior. SCSM 2018 (G. Meiselwitz, ed.), vol. 10913, pp. 431-444, Springer, 2018.

[4] W. Silva, C. Sacramento, E. Silva, A. C. Garcia, and S. B. L. Ferreira, "Health information, human factors and privacy issues in mobile health applications," in Proceedings of the 53rd Hawaii International Conference on System Sciences, pp. 3430-3438, ScholarSpace, 2020.

[5] C. Aiello, "Under armous says data breach affected about 150 million myfitnesspal accounts," 2018. Accessed November 30, 2020.

[6] A. Hern, "Fitness tracking app strava gives away location of secret us army bases," 2018. Accessed November 30, 2020.

[7] K. Masuch, M. Greve, and S. Trang, "Fitness first or safety first? examining adverse consequences of privacy seals in the event of a data breach," in Proceedings of the 54th Hawaii International Conference on System Sciences, ScholarSpace, 2021.

[8] S. Emerson, C. Heavin, and D. J. Power, "Workplace health promotion: Effects of an mhealth application on employee behavior and welness," in Proceedings of the 54th Hawaii International Conference on System Sciences, pp. 3419-3428, ScholarSpace, 2020.

[9] H. Paredes, D. Paulino, J. Barroso, C. Abrantes, I. Machado, and I. Silva, "Supervised physical exercise therapy of peripheral artery disease patients: M-health challenges and opportunities," in Proceedings of the 54th Hawaii International Conference on System Sciences, ScholarSpace, 2021. 